\title{
Mach Number Impact on Heat Flux and Pressure Distributions of a Hypersonic Flow over Combined Gap/Step Geometries
}

\author{
Paulo H. M. Leite and Wilson F. N. Santos \\ Combustion and Propulsion Laboratory (LCP), National Institute for Space Research (INPE) \\ Cachoeira Paulista-SP, 12630-000, Brazil
}

\begin{abstract}
A computational analysis of a hypersonic flow over a combined gap/step configuration at zero degree angle of attack, in chemical equilibrium and thermal non-equilibrium is presented in this work. Effects on pressure and heating loads due to changes on the freestream Mach number and on the step frontal-face height have been investigated by employing the Direct Simulation Monte Carlo (DSMC) method. The work focuses the attention of designers of hypersonic configurations on the fundamental parameter of surface discontinuity, which can have an important impact on even initial design. The analysis showed that heating and pressure loads increased with increasing not only the step height but also with the freestream Mach number. In addition, peak values for both loads took place at the vicinity of the step convex corner, a similar behavior observed for a forward-facing step configuration. It was also found that these loads for the gap/step configuration are slightly smaller than those for a forward-facing step.
\end{abstract}

Keywords: DSMC, hypersonic flow, rarefied flow, gap, forward-facing step, heating load.

PACS: 47.45.-n, 47.40.Ki, 47.45.-n

\section{INTRODUCTION}

It is generally recognized that the physical phenomena related to the problem of separation are very important in the design of hypersonic configurations. When separation occurs in high Mach number flows, changes in pressure distribution and heat transfer rate can have catastrophic effects in the vehicle surface. The presence of hot spots at separation and reattachment points changes the characteristics of the flow over the vehicle and may cause failure in the thermal protection system, as was evidenced by the tragic loss of the Space Shuttle Columbia in 2003. In general, separation occurs due to the interaction of external flows with various desired or undesired design features present on the vehicle surface, such as protuberances, notches, cavities, gaps, or steps.

In the current literature, there are extensive studies dealing with flows on cavities, gaps, or steps. In general, these research studies have been conducted in order to understand, among others, the physical aspects of a laminar or turbulent boundary layer in a subsonic, supersonic or hypersonic flow past to these types of surface discontinuity, characterized by a sudden change on the surface slope. The major interest in these research studies has gone into considering flows in the continuum regime. However, there is little understanding of the physical aspects of rarefied hypersonic flows past to these discontinuities related to the severe aerothermodynamic environment associated with a reentry vehicle.

In this scenario, Leite and Santos [1] have investigated forward-facing steps situated in a rarefied hypersonic flow by employing the DSMC method. The study was motivated by the interest in investigating the frontal-face height effect on the flowfield structure and on the aerodynamic surface properties in the transition flow regime. The computational results showed that changes in the step height contributed to significant modifications in the flowfield structure ahead the step. High pressure and heating loads were observed at the vicinity of the step frontal face as a consequence of the recirculation region ahead the step.

In the following, Paolicchi and Santos [2] have studied gaps situated in a rarefied hypersonic flow also by employing the DSMC method. The work was undertaken in an attempt to investigate the length-to-depth $(L / H)$ ratio effects on the flowfield structure. The primary emphasis was to examine the behavior of the primary properties, such as velocity, density, pressure and temperature, due to changes on the gap $L / H$ ratio. The analysis showed that the gap flow behavior in the transition flow regime differs from that found in the continuum flow regime, for the conditions investigated. It was found only one vortex for the $L / H$ ratio of $1,1 / 2,1 / 3$ and $1 / 4$. Conversely, in the continuum flow regime, the number of vortices inside the gap is approximately given by the amount $H / L$ [3].

Proceedings of the 29th International Symposium on Rarefied Gas Dynamics

AIP Conf. Proc. 1628, 176-184 (2014); doi: 10.1063/1.4902590

(C) 2014 AIP Publishing LLC 978-0-7354-1265-1/\$30.00 

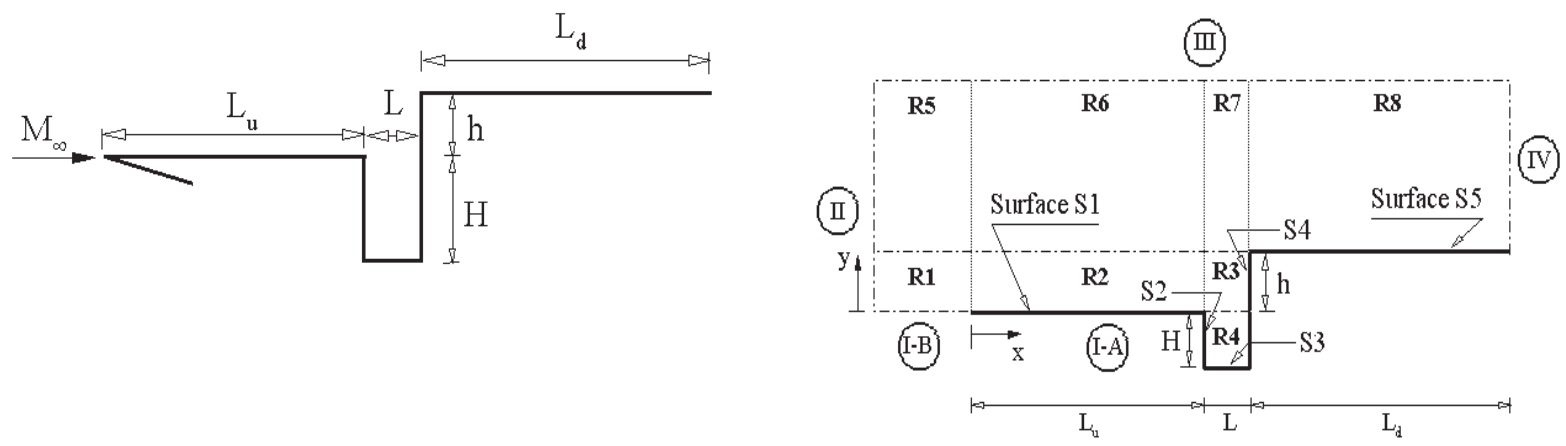

FIGURE 1. Drawing illustrating (a) the gap/step configuration and (b) the computational domain.

In the present account, effort is directed to expand the previous studies [1, 2] by investigating another surface discontinuity defined by the combination of a gap and a forward-facing step. This type of surface discontinuity, a combined gap/step, may occur in the windward surface of the Space Shuttle configuration, for instance. The windward surface is composed by a large number of thermal protection tiles. However, a misalignment between individual tiles may occur. Post flight measurements indicated tile mismatches were as large as $3 \mathrm{~mm}$ in step height and $4 \mathrm{~mm}$ in gap width [4]. Therefore, the misaligned tiles may constitute in a potential source in a heat flux and pressure rise on the surface or even though in a premature transition from laminar to turbulent flow. In this fashion, the primary goal is to provide a comprehensive description of the compressibility effects on pressure and heating loads for a family of gap/step configurations. Freestream flow conditions employed in the present calculations are those given in the previous work [1,2], and represent those experienced by a reentry vehicle at an altitude of $70 \mathrm{~km}$ with freestream Mach number of 5, 15, and 25. In these conditions, the degree of molecular non-equilibrium is such that the NavierStokes equations are inappropriate. Therefore, the DSMC method will be employed to calculate the hypersonic flow on the combined gap/step configuration.

\section{GEOMETRY DEFINITION}

In the present effort, the gap/step configuration is modeled by a flat plate with a gap of length $L$ and depth $H$, and a forward-facing step with height $h$. Figure 1(a) displays a schematic view of the model employed. The flat plate was selected by considering that the gap depth $H$ or the step height $h$ is much smaller than the nose radius $R$ of a reentry vehicle, i.e., $H / R \ll 1$ or $h / R \ll 1$. Therefore, the hypersonic flow over the flat plate with a gap/step may be representative of the hypersonic flow over a gap/step configuration located on the surface of a reentry vehicle.

Based on Fig. 1(a), $M_{\infty}$ represents the freestream Mach number, $H$ is the gap depth, $h$ is the step height, $L_{u}$ the length of the gap/step upstream surface, $L$ the length of the gap, and $L_{d}$ the length of the gap/step downstream surface. It was assumed a gap length $L$ of $3 \mathrm{~mm}$ and a gap depth $H$ of $3 \mathrm{~mm}$, which correspond to a length-to-depth ratio, $L / H$, of 1 . Furthermore, the step height $h$ investigated was defined as being 3, 6, and $9 \mathrm{~mm}$, which correspond to the dimensionless height $h^{*}\left(\equiv h / \lambda_{\infty}\right)$ of 3.23,6.46, and 9.69, respectively, where $\lambda_{\infty}$ is the freestream mean free path. Moreover, it was defined $L_{u} / \lambda_{\infty}$ of 50 and $L_{d} / \lambda_{\infty}$ of 50 . It was considered that the flat plate is infinitely long but only the total length $L_{u}+L+L_{d}$ is investigated.

\section{FREESTREAM AND FLOW CONDITIONS}

Freestream flow conditions used for the numerical simulation of flow past to the combined gap/step configuration are those given by Leite and Santos [1] and summarized in Table 1, and the gas properties [5] are shown in Table 2.

Referring to Tables 1 and $2, T_{\infty}, p_{\infty}, \rho_{\infty}, n_{\infty}, \mu_{\infty}, \lambda_{\infty}$, and $U_{\infty}$ stand respectively for temperature, pressure, density, number density, viscosity, mean free path and velocity, and $\chi, m, d$ and $\omega$ account respectively for mole fraction, molecular mass, molecular diameter, and viscosity index for $\mathrm{O}_{2}$ and $\mathrm{N}_{2}$. 
TABLE 1. Freestream flow conditions [1].

\begin{tabular}{cccccccc}
\hline Altitude (km) & $T_{\infty}(\mathbf{K})$ & $p_{\infty}\left(\mathbf{N} / \mathbf{m}^{2}\right)$ & $\rho_{\infty}\left(\mathbf{k g} / \mathbf{m}^{3}\right)$ & $n_{\infty}\left(\mathbf{m}^{-3}\right)$ & $\mu_{\infty}\left(\mathbf{N s} / \mathbf{m}^{2}\right)$ & $\lambda_{\infty}(\mathbf{m})$ & $U_{\infty}(\mathbf{m} / \mathbf{s})$ \\
\hline 70 & 220.0 & 5.582 & $8.753 \times 10^{-5}$ & $1.8209 \times 10^{21}$ & $1.455 \times 10^{-5}$ & $9.03 \times 10^{-4}$ & $1485 / 4528 / 7546$ \\
\hline
\end{tabular}

TABLE 2. Gas properties [5].

\begin{tabular}{cccccccc}
\hline$\chi\left(\mathbf{O}_{2}\right)$ & $m(\mathbf{k g})\left(\mathbf{O}_{2}\right)$ & $d(\mathbf{m})\left(\mathbf{O}_{2}\right)$ & $\omega\left(\mathbf{O}_{2}\right)$ & $\chi\left(\mathbf{N}_{2}\right)$ & $m(\mathbf{k g})\left(\mathbf{N}_{2}\right)$ & $d(\mathbf{m})\left(\mathbf{N}_{2}\right)$ & $\omega\left(\mathbf{N}_{2}\right)$ \\
\hline 0.237 & $5.312 \times 10^{-26}$ & $4.01 \times 10^{-10}$ & 0.77 & 0.763 & $4.650 \times 10^{-26}$ & $4.11 \times 10^{-10}$ & 0.74 \\
\hline
\end{tabular}

The freestream velocity $U_{\infty}$, assumed to be constant at 1485,4528 , and $7546 \mathrm{~m} / \mathrm{s}$, corresponds to freestream Mach numbers $M_{\infty}$ of 5, 15, and 25, respectively. The translational and vibrational temperatures in the freestream are in equilibrium at $220 \mathrm{~K}$, and the gap/step surface has a constant wall temperature $T_{w}$ of $880 \mathrm{~K}$ for all cases considered.

The overall Knudsen number $K n$ is defined as the ratio of the molecular mean free path $\lambda$ in the gas to a characteristic dimension of the flowfield. By assuming the step frontal-face height $h$ as the characteristic length, the Knudsen number $K n_{h}$ corresponds to $0.3095,0.1548$ and 0.1032 for dimensionless step height $h^{*}$ of $3.23,6.46$ and 9.69 , respectively. Finally, the Reynolds number $R e_{h}$, also based on the frontal-face height $h$ and on conditions in the undisturbed stream, is in the range from 27 to 409 for the step height investigated.

\section{COMPUTATIONAL METHOD AND PROCEDURE}

It is well know that neither the continuum flow equations nor the collisionless flow equations are valid to predict aerothermodynamics characteristics throughout the transitional flow regime. Nowadays, the Direct Simulation Monte Carlo (DSMC) method, developed by Bird [5], is one of the most accurate and credible procedures for computing aerothermodynamics in the transitional flow regime.

The DSMC method simulates real gas flows with various physical processes by means of a huge number of modeling particles, each of which is a typical representative of great number of real gas molecules. In the DSMC method, the state of the particles is stored and modified with time as the particles move, collide, and undergo boundary interactions in simulated physical space.

In the present account, the molecular collisions are modeled by using the variable hard sphere (VHS) molecular model [6] and the no time counter (NTC) collision sampling technique [7]. The energy exchange between kinetic and internal modes is controlled by the Borgnakke-Larsen statistical model [8]. Simulations are performed by using a non-reacting gas model consisting of two chemical species, $\mathrm{N}_{2}$ and $\mathrm{O}_{2}$. Energy exchanges between the translation and internal modes, rotation and vibration, are considered. Relaxation collision numbers are obtained in a collision energy-based procedure as suggested by Boyd [9] for rotation and by Bird [10] for vibration. For a given collision, the probabilities are designated by the inverse of the relaxation numbers, which correspond to the number of collisions necessary, on average, for a molecule to relax.

In order to implement the particle-particle collisions, the flowfield around the gap/step configuration is divided into an arbitrary number of regions, which are subdivided into computational cells. The cells are further subdivided into subcells, two subcells/cell in each coordinate direction. The cell provides a reference for the sampling of the macroscopic gas properties, such as density, velocity, pressure, temperature, etc., while the subcell is used in order to select collision partners for the establishment of the collision rate. Therefore, the physical space network is used to facilitate the choice of molecules for collisions and for the sampling of the macroscopic flow properties.

The computational domain used for the simulation is made large enough so that disturbances from the gap/step configuration do not reach the upstream and side boundaries, where freestream conditions are specified. A schematic view of the computational domain is demonstrated in Fig. 1(b). According to this figure, side I-A is defined by the gap/step surface. Diffuse reflection with complete thermal accommodation was defined as the condition applied to this side. Side I-B represents a plane of symmetry, where all flow gradients normal to the plane are zero. At the molecular level, this plane is equivalent to a specular reflecting boundary. Sides II and III are the freestream side through which simulated molecules enter and exit. Side II was positioned at $5 \lambda_{\infty}$ upstream of the flat-plate leading edge for all cases investigated. Side III, for Mach number cases of 5 and 15, was defined at $45 \lambda_{\infty}, 45 \lambda_{\infty}$, and $52 \lambda_{\infty}$ above surface S5 for frontal-face height $h^{*}$ of 3.23, 6.46, and 9.69, respectively. For Mach number case of 25, side III was defined at 30 $\lambda_{\infty}$, 
TABLE 3. Region dimensions $(x \times y)$ and number of cells [ $x$-direction $\times y$-direction] as a function of the step height $h^{*}$ for Mach number of 25 .

\begin{tabular}{lccc}
\hline & $h^{*}=\mathbf{3 . 2 3}$ & $h^{*}=\mathbf{6 . 4 6}$ & $h^{*}=\mathbf{9 . 6 9}$ \\
\hline R1 & $\left(5 \lambda_{\infty} \times 3.23 \lambda_{\infty}\right)[20 \times 10]$ & $\left(5 \lambda_{\infty} \times 6.46 \lambda_{\infty}\right)[20 \times 10]$ & $\left(5 \lambda_{\infty} \times 9.69 \lambda_{\infty}\right)[20 \times 10]$ \\
R2 & $\left(50 \lambda_{\infty} \times 3.23 \lambda_{\infty}\right)[130 \times 40]$ & $\left(50 \lambda_{\infty} \times 6.46 \lambda_{\infty}\right)[130 \times 40]$ & $\left(50 \lambda_{\infty} \times 9.69 \lambda_{\infty}\right][130 \times 40]$ \\
R3 & $\left(3.23 \lambda_{\infty} \times 3.23 \lambda_{\infty}\right)[40 \times 100]$ & $\left(3.23 \lambda_{\infty} \times 6.46 \lambda_{\infty}\right)[40 \times 120]$ & $\left(3.23 \lambda_{\infty} \times 9.69 \lambda_{\infty}\right)[40 \times 140]$ \\
R4 & $\left(3.23 \lambda_{\infty} \times 3.23 \lambda_{\infty}\right)[40 \times 35]$ & $\left(3.23 \lambda_{\infty} \times 3.23 \lambda_{\infty}\right)[40 \times 35]$ & $\left(3.23 \lambda_{\infty} \times 3.23 \lambda_{\infty}\right)[40 \times 35]$ \\
R5 & $\left(5 \lambda_{\infty} \times 30 \lambda_{\infty}\right)[20 \times 60]$ & $\left(5 \lambda_{\infty} \times 34 \lambda_{\infty}\right)[20 \times 60]$ & $\left(5 \lambda_{\infty} \times 42 \lambda_{\infty}\right)[20 \times 60]$ \\
R6 & $\left(50 \lambda_{\infty} \times 30 \lambda_{\infty}\right)[110 \times 60]$ & $\left(50 \lambda_{\infty} \times 34 \lambda_{\infty}\right)[110 \times 60]$ & $\left(50 \lambda_{\infty} \times 42 \lambda_{\infty}\right)[110 \times 60]$ \\
R7 & $\left(3.23 \lambda_{\infty} \times 30 \lambda_{\infty}\right)[20 \times 60]$ & $\left(3.23 \lambda_{\infty} \times 34 \lambda_{\infty}\right)[20 \times 60]$ & $\left(3.23 \lambda_{\infty} \times 42 \lambda_{\infty}\right)[20 \times 60]$ \\
R8 & $\left(50 \lambda_{\infty} \times 30 \lambda_{\infty}\right)[130 \times 70]$ & $\left(50 \lambda_{\infty} \times 34 \lambda_{\infty}\right)[130 \times 70]$ & $\left(50 \lambda_{\infty} \times 42 \lambda_{\infty}\right)[130 \times 70]$ \\
\hline
\end{tabular}

$34 \lambda_{\infty}$, and $42 \lambda_{\infty}$ for $h^{*}$ of $3.23,6.46$, and 9.69 , respectively. Finally, the flow at the downstream outflow boundary, side IV, is predominantly supersonic and vacuum condition is specified [5]. Basically, at this boundary, simulated molecules can only exit. Nevertheless, it is important to mention that, close to the wall, molecules may not be moving at supersonic speed. As a result, in this subsonic region close to the wall, there is an interaction between the flow and the downstream boundary. However, the extent of the upstream effect of this boundary condition can be determined by changing the length of surface S5. For the conditions investigated in the present account, the upstream disturbance is in the range from 6 to $10 \lambda_{\infty}[1]$.

DSMC results depend on the cell size chosen, on the time step as well as on the number of particles per computational cell. The linear dimensions of the cells should be small in comparison with the length scale of the macroscopic flow gradients normal to the streamwise directions, which means that the cell dimensions should be the order of or smaller than the local mean free path [5]. The time step should be chosen to be sufficiently small in comparison with the local mean collision time [5]. Finally, the number of simulated particles has to be large enough to make statistical correlations between particles insignificant. It is advisable that each cell be populated with a minimum number of molecules, typically around twenty [5].

A grid independence study was made with three different structured meshes - coarse, standard and fine - in each coordinate direction. The effect of altering the cell size in the $x$ - and $y$-directions was investigated for a coarse and fine grids with, respectively, 50\% less and 100\% more cells based on the standard grid. Table 3 summarizes the main characteristics for the standard grid related to eight regions (R1 to R8 in Fig. 1(b)) for the frontal-face height $h^{*}$ of 3.23, 6.46, and 9.69, and Mach number of 25. It is important to remark the relative cell size to local mean free path for the standard grid ranged from 0.061 to around 1.0 (for cells close to side III).

The effect (not shown) of changing the cell size in both $x$ - and $y$-directions on the heat transfer, pressure and skin friction coefficients was rather insensitive to the range of cell spacing considered, indicating that the standard grids, with a total of 28900, 29700, and 30500 cells are essentially grid independent.

\section{COMPUTATIONAL RESULTS AND DISCUSSION}

Before proceeding with the analysis of pressure and heating loads, it proves instructive to present first the flow topology around the gap/step configuration. In doing so, density ratio, $\rho / \rho_{\infty}$, contours with streamline traces around the gap/step configuration are demonstrated in Fig. 2 for $M_{\infty}$ of 25, a gap $L / H$ ratio of 1 and step height $h^{*}$ of 3.23, 6.46, and 9.69. In this group of plots, dimensionless height $Y$ stands for the height $y$ normalized by the freestream mean free path $\lambda_{\infty}$, and the dimensionless length $X$ refers to the length $x$ also normalized by the $\lambda_{\infty}$. It may be recognized from this group of plots that density ratio increases inside the gap by increasing the frontal-face height $h^{*}$, for the conditions investigated. This is in contrast to the gap (without step) behavior investigated previously [2]. Consequently, the step frontal face acts as a barrier in the sense that part of the flow enters into the gap. In addition, it is clearly noticed that the flow inside the gap is characterized by the appearance of recirculation regions. The streamline pattern shows that the flow is characterized by only one vortex for the $h^{*}=3.23$ case, and two vortices for the other two cases, $h^{*}$ of 6.46 and 9.69. A similar behavior is observed for the other two freestream Mach number $M_{\infty}$ of 5 and 15. 

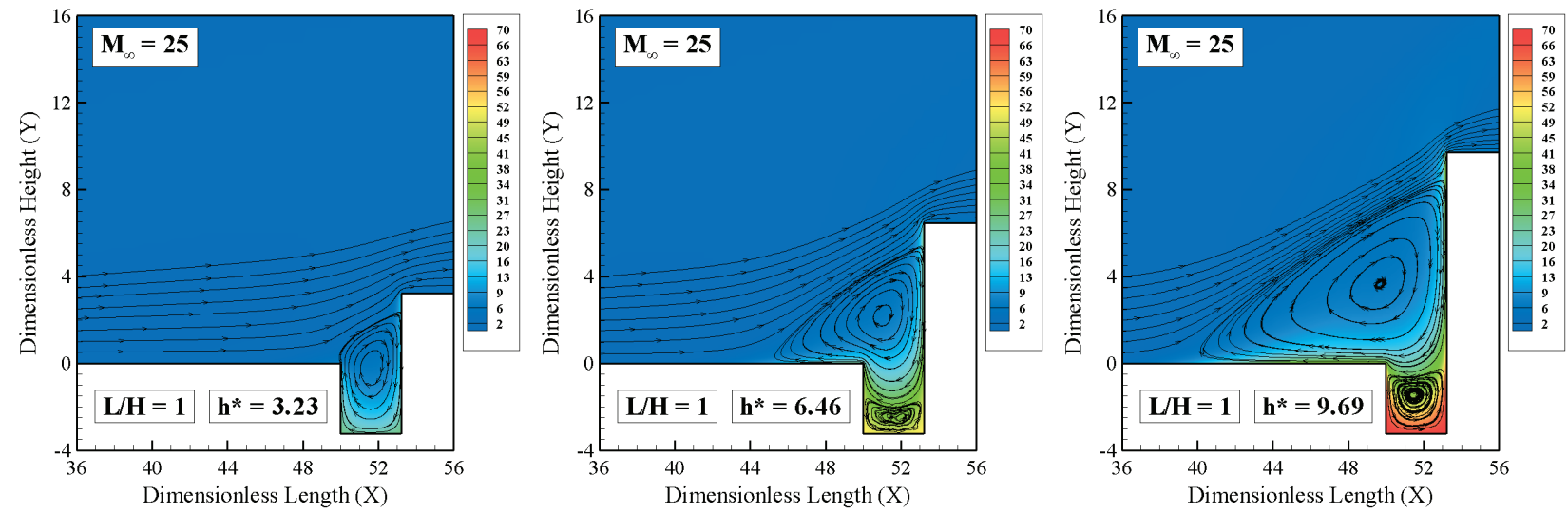

FIGURE 2. Density ratio $\left(\rho / \rho_{\infty}\right)$ contour maps along with streamline traces at the vicinity of the gap/step configuration for a gap $L / H$ ratio of 1 and dimensionless step height $h^{*}$ of (a) 3.23, (b) 6.46, and (c) 9.69 .

\section{Pressure Coefficient}

The pressure $p_{w}$ acting on the body surface is calculated by the sum of the normal momentum fluxes of both incident and reflected molecules at each time step on the body surface. The pressure coefficient $C_{p}$ is defined as being $p_{w^{-}} p_{\infty}$ normalized by the freestream dynamic pressure, $\frac{1}{2} \rho_{\infty} U_{\infty}^{2}$.

Distributions of pressure coefficient $C_{p}$ along surfaces S1 and S5 are illustrated in Figs. 3 and 4 for $h^{*}$ of 3.23 and 9.69, respectively, parameterized by the freestream Mach number. As a basis of comparison, the pressure coefficient $C_{p}$ for the flat-plate case, step [1] and gap [2] is also illustrated in these plots. In addition, results for the gap/step case defined by $h^{*}$ of 6.46 are intermediate to cases presented in these plots and they will not be shown.

According to this set of plots, it is noted that the pressure coefficient behavior for the gap/step configuration follows the same trend as that shown for the forward-facing step case in the sense that the maximum values for $C_{p}$ increases not only by increasing the step frontal face $h^{*}$ but also by increasing the freestream Mach number $M_{\infty}$. In addition, peak values for the gap/step configuration are smaller than those for the forward-facing step case. This behavior is explained by the fact that, for the forward-facing step, the number flux (not shown), i.e., the number of molecules colliding to the body surface by unit time and unit area, dramatically increases at the vicinity of the step concave corner, i.e., at the step base. In contrast, for the gap/step configuration, due to the flow expansion around surface$\mathrm{S} 1 /$ surface-S2 junction, the number flux is lower than that for the forward-facing step. As a result, pressure coefficient
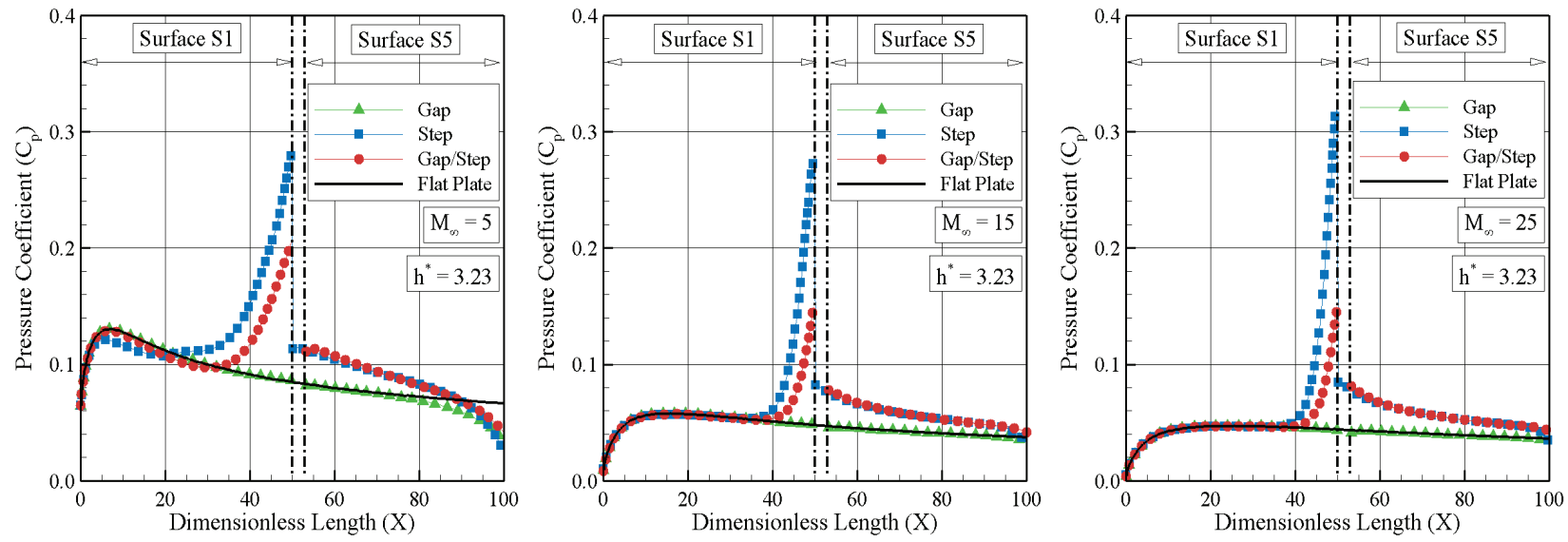

FIGURE 3. Distribution of pressure coefficient $C_{p}$ along surfaces S1 and S5 for the $h^{*}=3.23$ case and freestream Mach number of 5 (left), 15 (middle) and 25 (right). 

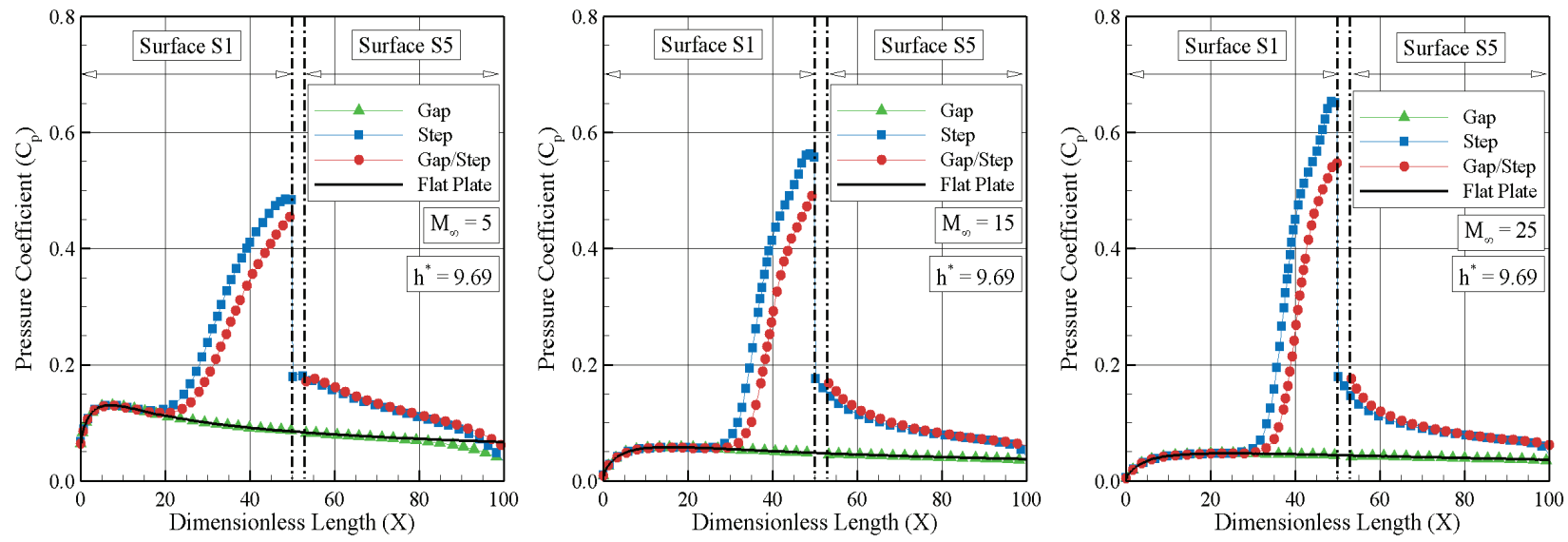

FIGURE 4. Distribution of pressure coefficient $C_{p}$ along surfaces S1 and S5 for the $h^{*}=9.69$ case and freestream Mach number of 5 (left), 15 (middle) and 25 (right).
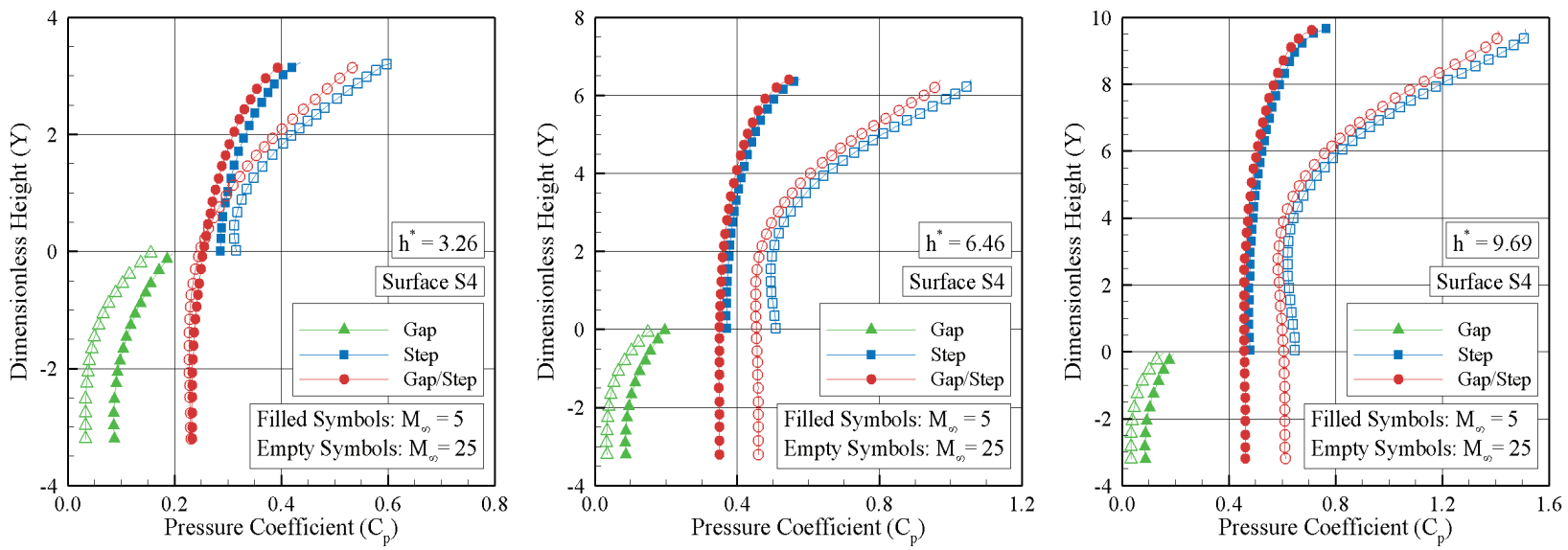

FIGURE 5. Distribution of pressure coefficient $C_{p}$ along surface $\mathrm{S} 4$ for dimensionless step height $h^{*}$ of 3.23 (left), 6.46 (middle) and 9.69 (right).

for the gap/step configuration is lower than that for forward-facing step at the vicinity of the step frontal face. It should be mentioned in this context that density, as well as pressure, reaches maximum values at the step concave corner for the forward-facing step. Conversely, for the gap/step configuration, the maximum values for density and pressure are observed inside the gap, more precisely at the vicinity of the gap bottom surface, as illustrated in Fig. 2. It should be also remarked that wall pressure $p_{w}$ was normalized by $\frac{1}{2} \rho_{\infty} U_{\infty}^{2}$, and $U_{\infty}$ differs from one to another Mach number case.

Distributions of pressure coefficient $C_{p}$ along surface S4 is displayed in Fig. 5 parameterized by the step height $h^{*}$. In this group of plots, filled and empty symbols correspond to Mach number of 5 and 25, respectively. Results for Mach number of 15 are intermediate to the cases shown and they will not be presented. According to this group of plots, it is clearly seen that the pressure coefficient $C_{p}$ for the gap/step configuration follows the same behavior as that shown for the forward-facing step case in the sense that the maximum values for $C_{p}$ increases not only by increasing the step frontal face $h^{*}$ but also by increasing the freestream Mach number $M_{\infty}$. Similar to the forward-facing step case, peak values for the gap/step configuration take place at the vicinity of the step convex corner, i.e., close to the surface-S4/surface-S5 junction. In addition, it is also observed that peak values on the step frontal face are larger than those on surface $\mathrm{S} 1$.

As a matter of comparison, for Mach number of 25, maximum values for $C_{p}$ on the frontal face are around 0.55 , 0.97 and 1.41 for height $h^{*}$ of 3.23, 6,46 and 9.69, respectively. Conversely, the maximum value of $C_{p}$ for the flat-plate 
case, i.e., a flat plate without a gap/step configuration, is around 0.047 at section $X=25.5$ on surface $\mathrm{S} 1$. Therefore, $C_{p}$ of $0.55,0.97$ and 1.41 correspond respectively to $11.7,20.6$ and 30.0 times the peak value for the flat-plate case, which corresponds to a smooth surface.

\section{Heat Transfer Coefficient}

The heat flux $q_{w}$ to the body surface is calculated by the net energy flux of the molecules impinging on the surface. The net heat flux $q_{w}$ is related to the sum of the translational, rotational and vibrational energies of both incident and reflected molecules. A flux is regarded as positive if it is directed toward the body surface. The heat flux is normalized by $\frac{1}{2} \rho_{\infty} U_{\infty}^{3}$ and presented in terms of heat transfer coefficient $C_{h}$.

Distributions of heat transfer coefficient $C_{h}$ along surfaces S1 and S5 are demonstrated in Figs. 6 and 7 for $h^{*}$ of 3.23 and 9.69, respectively. Again, for comparison purpose, the heat transfer coefficient $C_{h}$ for the flat-plate case, step [1] and gap [2] is also illustrated in these figures. In addition, due to the large range of variation for the heat transfer coefficient $C_{h}$, the scale in the plots for the $h^{*}=3.23$ case differs from that for the $h^{*}=9.69$ case.

According to Figs. 6 and 7, the heat transfer coefficient $C_{h}$ for the gap/step configuration follows the same behavior presented by the flat-plate case close to the sharp leading edge, region unaffected not only by the presence of the
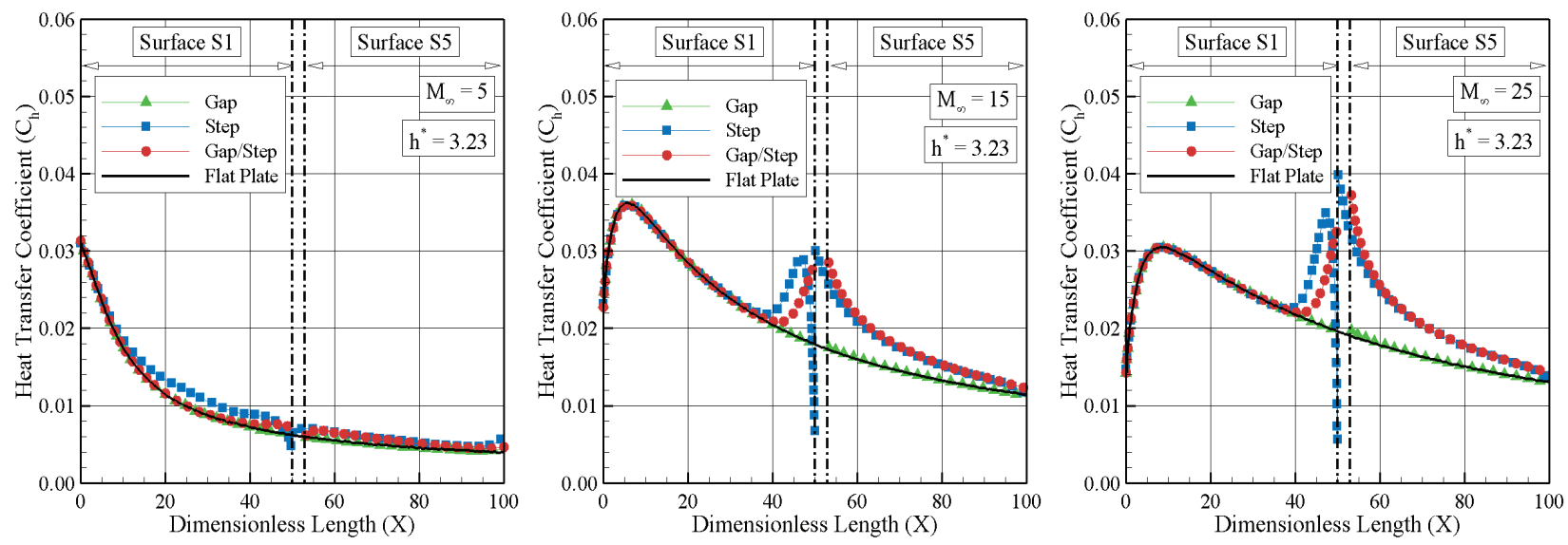

FIGURE 6. Distribution of heat transfer coefficient $C_{h}$ along surfaces $\mathrm{S} 1$ and S5 for the $h^{*}=3.23$ case and freestream Mach number of 5 (left), 15 (middle) and 25 (right).
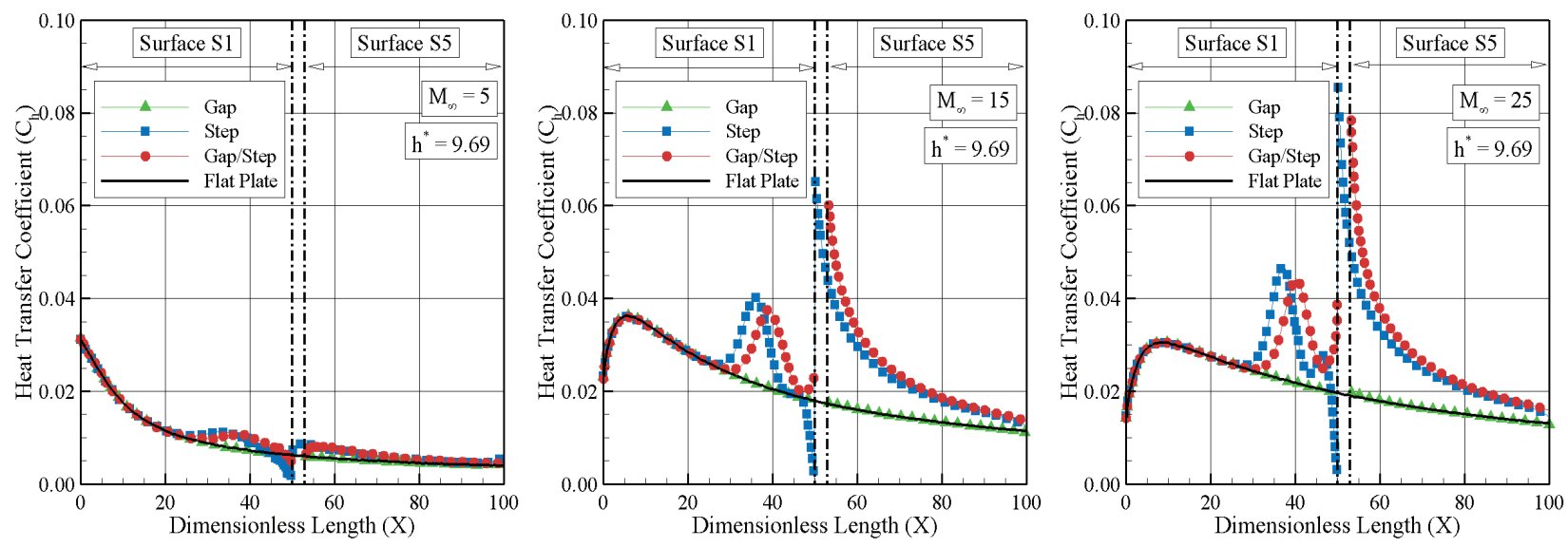

FIGURE 7. Distribution of heat transfer coefficient $C_{h}$ along surfaces S1 and S5 for the $h^{*}=9.69$ case and freestream Mach number of 5 (left), 15 (middle) and 25 (right). 

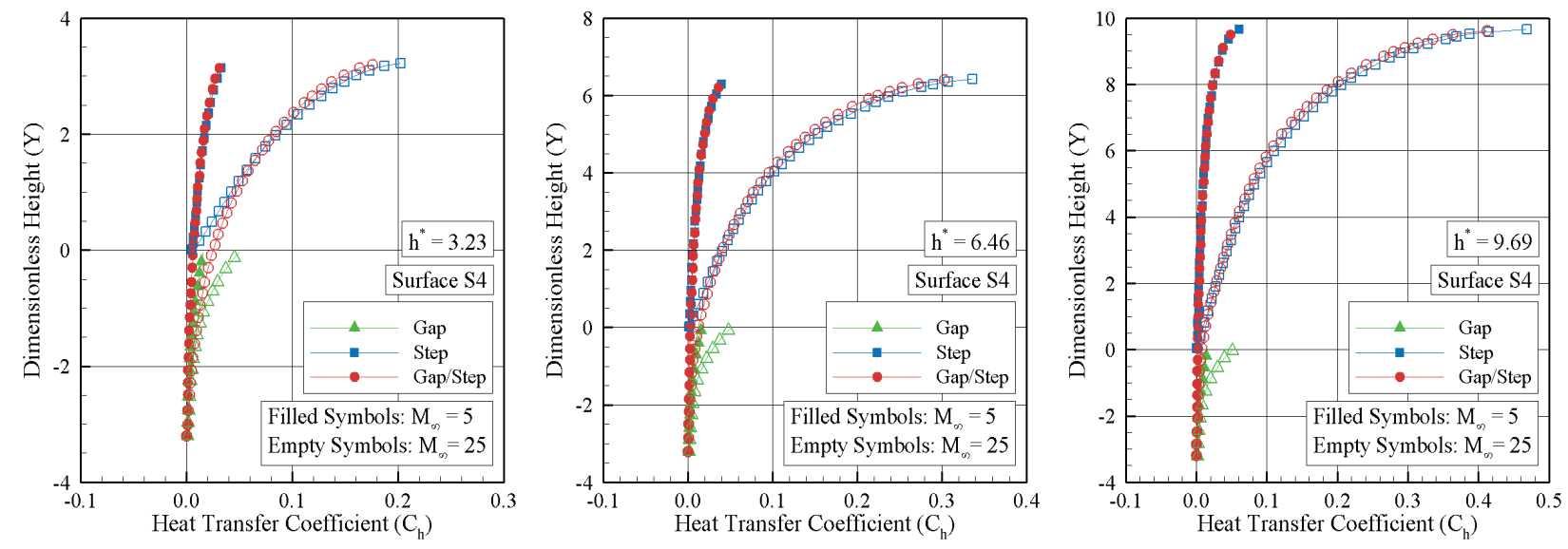

FIGURE 8. Heat transfer coefficient $C_{h}$ along surface $\mathrm{S} 4$ for dimensionless step height $h^{*}$ of 3.23 (left), 6.46 (middle) and 9.69 (right).

gap/step configuration but also by the gap or step alone. Further downstream along surface S1, the heat transfer coefficient $C_{h}$ significantly increases and reaches peak values at the vicinity of the surface S1/surface S2 junction. In contrast, after reaching peak values, $C_{h}$ for the forward-facing step decreases to almost zero at the step base. Along surface S5, the heat transfer coefficient $C_{h}$ presents a maximum value at the step convex corner and then decreases downstream along the surface, basically reaching the values observed for the flat-plate case.

Along surface S4, Fig. 8, the heat transfer coefficient increases monotonically, from zero at surface-S3/surface-S4 junction to a maximum value near the step convex corner. The maximum value depends not only on the frontal-face height $h$ but also on the freestream Mach number. It is quite apparent that this significant increase in the heat transfer coefficient is due to the flow reattachment zone. It is important to mention that peak values observed for the heat transfer coefficient on the step frontal-face surface is one order of magnitude larger than those observed on surface $\mathrm{S} 1$. The reason for that is because the step face acts as a stagnation region for the reattaching shear layer. As a base of comparison, for freestream Mach number of 25, the maximum values for $C_{h}$ are around 0.18, 0.30 and 0.41 for height $h^{*}$ of 3.23, 6,46 and 9.69, respectively. In contrast, the $C_{h}$ for the flat-plate case, i.e., a flat plate without discontinuities, is around 0.030 at section $X=9.1$ on surface S1. Therefore, $C_{h}$ of $0.18,0.30$ and 0.41 correspond respectively to 6.0, 10.0 and 13.7 times the peak value for the flat-plate case.

\section{CONCLUDING REMARKS}

A computational analysis has been carried out in order to investigate a rarefied hypersonic flow over a gap/step configuration by employing the Direct Simulation Monte Carlo method. In the present account, the gap/step configuration models a misalignment observed between individual tiles in the thermal protection system of reentry vehicles, such as the Space Shuttle. The main focus was to analyze the effects of the freestream Mach number and the step frontal-face height on heating and pressure loads. Both effects were investigated for a representative range of parameters. The freestream Mach number varied from 5 to 25, and the step frontal-face height ranged from 3 to 9 millimeters, which corresponded overall Knudsen numbers from 0.3095 to 0.1032 .

The analysis showed that changes on the freestream Mach number affected the heating and pressure loads, on the step upstream surface and on frontal face, for the range of parameters investigated. It was also found that heating and pressure loads increased with increasing not only the freestream Mach number but also with increasing the step frontal face. It was found that these loads exhibited maximum values on the step face, more precisely at the vicinity of the step shoulder. In addition, these loads are much higher than the maximum values found for a smooth surface, i.e., a flat plate without a gap/step configuration. Consequently, in a hypersonic vehicle design, heating and pressure loads become important if discontinuities, such as a gap/step configuration, are present in the vehicle surface. 


\section{REFERENCES}

1. P. H. M. Leite, W. F. N. Santos, 42nd AIAA Thermophysics Conference, AIAA Paper 2011-3310, Honolulu, Hawaii (2011).

2. L. T. L. C. Paolicchi, W. F. N. Santos, 44th AIAA Thermophysics Conference, AIAA Paper 2013-2789, San Diego, CA (2013).

3. J. L. Everhart, S. J. Alter, N. R. Merski, W. A. Wood, R. K. Prabhu, 44th AIAA Aerospace Sciences Meeting and Exhibit, AIAA Paper 2006-0185, Reno, NV (2006).

4. D. M. Smith, D. N. Petley, C. L. W. Edwards, A. B. Patten, 21st AIAA Aerospace Sciences Meeting and Exhibit, AIAA Paper 1983-0120, Reno, NV (1983).

5. G. A. Bird, Molecular Gas Dynamics and the Direct Simulation of Gas Flows, Oxford University Press, England, UK, 1994.

6. G. A. Bird, Progress in Astronautics and Aeronautics: Rarefied gas Dynamics, AIAA New York, 74:239-255, 1981.

7. G. A. Bird, Rarefied Gas Dynamics: Theoretical and Computational Techniques, AIAA, New York, 118:374-395, 1989.

8. C. Borgnakke, P. S. Larsen, Journal of computational Physics, 18, 405-420 (1975).

9. I. D. Boyd, AIAA Journal, 28:1997-1999, 1998.

10. G. A. Bird, 26th International Symposium on Rarefied Gas Dynamics, American Institute of Physics, 245-250, 2009. 
AIP Conference Proceedings is copyrighted by AIP Publishing LLC (AIP). Reuse of AIP content is subject to the terms at: http://scitation.aip.org/termsconditions. For more information, see http://publishing.aip.org/authors/rights-and-permissions. 